\title{
Effectiveness and Safety of Brivaracetam in Children
}

\author{
Vykuntaraju K. Gowda ${ }^{1}$ (D) Balamurugan Nagarajan ${ }^{1} \cdot$ Sanjay K. Shivappa ${ }^{2} \cdot$ Naveen Benakappa $^{2}$
}

Received: 27 December 2020 / Accepted: 5 February 2021/ Published online: 12 February 2021

(C) Dr. K C Chaudhuri Foundation 2021

To the Editor: More than 25\% children with seizures have refractory epilepsy [1]. Brivaracetam is a United States Food and Drug Administration (USFDA) approved antiepileptic drug (AED) indicated in patients older than $4 \mathrm{y}$ [2]. In this retrospective study, we assessed the effectiveness and safety of Brivaracetam in children with refractory epilepsy below 18 y of age, between September 2018 and August 2019. Out of total 38 patients, 25 were males. Mean age was 5.9 y. Various epilepsy syndromes were West syndrome (6); LennoxGastaut syndrome (LGS) (8); Dravet syndrome (2); and tuberous sclerosis (TS) (3). The average dose of Brivaracetam used was $2 \mathrm{mg} / \mathrm{kg} /$ body weight.

Decrease in seizure frequency was more than $50 \%$ in 14/38 (36\%) with $4(10 \%)$ of them seizure-free, no change in 18 (47\%), less than $50 \%$ in 1, and $2(5.2 \%)$ showed worsening of seizures. More than $50 \%$ reduction noted in West syndrome (3), LGS (5), TS (2). Seizure-free in 3 cases of unknown etiology and 1 case of West syndrome. Eleven (55\%) of them had more than 50\% seizure reduction in Levetiracetam (LEV) switch cases. The adverse effects were drowsiness and mood swings in 1 child each. Two out of the 4 patients reported improvement in behavior in LEV switch cases. The duration of follow-up was $12 \mathrm{mo}$, and during this period, no change in effectiveness was noted in those who responded.

Brivaracetam has 15 - to 30 -fold high affinity for synaptic vesicle $2 \mathrm{~A}$ than LEV. It has high lipid solubility, rapid brain penetration, and broad-spectrum antiepileptic activity [2]. The safety and tolerability profile in children are similar to adults
$[2,3]$. We noted greater than $50 \%$ seizure reduction in $36 \%$, seizure-free in $10 \%$, unchanged seizure frequency in $47 \%$, increased seizure frequency in $5.2 \%$ compared to $44 \%, 17 \%$, $38 \%$, and $18 \%$, respectively reported in adults [4]. To conclude, Brivaracetam adjunctive treatment is well tolerated, safe, and effective in children.

\section{Declarations}

Conflict of Interest None.

\section{References}

1. Strzelczyk A, Griebel C, Lux W, Rosenow F, Reese JP. Burden of severely drug-refractory epilepsy: a comparative longitudinal evaluation of mortality, morbidity, resource use, and cost using German health insurance data. Front Neurol. 2017;8:712.

2. UCB, Brussels, Belgium. Briviact US Prescribing information 2018. Available at: https://www.briviact.com/briviact-PI.pdf. Accessed 25 January 2020.

3. Liu E, Dilley D, McDonough B, Stockis A, Daniels T. Safety and tolerability of adjunctive brivaracetam in pediatric patients $<16$ years with epilepsy: an open-label trial. Pediatr Drugs. 2019;21:291-301.

4. Menzler K, Mross PM, Rosenow F, et al. First clinical post marketing experiences in the treatment of epilepsies with Brivaracetam: a retrospective observational multicenter study. BMJ Open. 2019;9(11):e030746.

Publisher's Note Springer Nature remains neutral with regard to jurisdictional claims in published maps and institutional affiliations.
Vykuntaraju K. Gowda

drknvraju08@gmail.com

1 Department of Pediatric Neurology, Indira Gandhi Institute of Child Health, Bengaluru, Karnataka 560029, India

2 Department of Pediatric Medicine, Indira Gandhi Institute of Child Health, Bengaluru, Karnataka, India 